\title{
Optimization of Video Streaming through Bufferbloat Mitigation using Hybrid FQ-CoDel Algorithm
}

\author{
Vimaladevi V. \\ PG Scholar, Dept. of Computer Science \\ IFET College of Engineering \\ Villupuram, India
}

\author{
Manju Bala P. \\ Associate Professor, Dept. of Computer Science \\ IFET College of Engineering \\ Villupuram, India
}

\begin{abstract}
The major traffic in today's internet is entirely filled with video data with increased use of Video on-Demand systems. Quality Of internet video has a great impact on user engagement. Video encoding and streaming over wireless networks become a big concern. Real time video service requires low end-to-end delay and the major concern is to reduce the queuing delay which has high impact on the video quality. Bufferbloat is one of the main reasons for experiencing high queue latency at the intermediate nodes. Optimization of buffer size in the network nodes is never practiced, though several AQM were used for reducing the queue latency. In this work, Hybrid FQ-CoDel mechanism is proposed which is the combination of both CoDel and Adaptive CoDel AQM mechanism with fair queuing for the reduction of queuing delay and to optimize video transmission. Here the Adaptive CoDel is targeted for video traffic data and on the other hand the CoDel is used for other low traffic network flow. The mechanisms of network classifier and the scheduler is used to achieve fairness in the network queuing. Hybrid FQ-CoDeL is used for effective reduction of the queuing delay and to increase the network bandwidth utilization for video streams which will result in better quality of video.
\end{abstract}

\section{General Terms}

Computer Network, Network Delay, Control Mechanism.

\section{Keywords}

Bufferbloat, CoDel, Adaptive CoDel, Queuing Delay.

\section{INTRODUCTION}

The major traffic in today's Internet is Video Streams. With the increasing amount of data and video traffic in the Internet requires the network devices such as routers and other end devices to be capable of handling multiple Gigabit connections at given time. Since video traffic is delay sensitive, the End-toend Latency between the network devices is an important metric to be concerned with. This end-to-end latency can be classified as three components: transmission delay, propagation delay and queuing delay. Of these queuing delay is the main cause of uncertainty which varies frequently depending upon the buffer size of the network devices [1].

The queuing delay is the time a job waits in a queue until it can be executed. It is a key component of network delay. Mostly packets arrive at a router, where they have to be processed and transmitted. A router can only process one packet at a time. If packets arrive faster than the router can process them (such as in a burst transmission) the router puts them into the queue (also called the buffer) until it can get around to transmitting them. Delay can also vary from packet to packet so averages and statistics are usually generated when measuring and evaluating queuing delay. The maximum queuing delay is proportional to buffer size.
An optimization in buffer size is required to enhance the QoS parameters such as queuing delay, link utilization, end-to-end throughput and packet loss. For the enhanced performance, high throughput is necessary and to reduce the queuing delay the availability of data in the buffer is to be made less as possible [6]. While the former needs large buffer size in order to increase the sending rate, the latter case requires reduction in buffer size to prevent the problem of bufferbloat [2].

The data traffic over the network requires minimum queuing delay, which can be obtained through one of the prominent AQM mechanisms called Controlled Delay (CoDel) [3]. But the drawback in CoDel is that, it does not support real-time video streaming since it uses fixed and uniform target value for processing. Adaptive CoDel is used for real-time video streaming to mitigate bufferbloat and to improve the QoS parameters [4]. Providing fairness which is not possible through CoDel is ensured by Fair queuing CoDel (FQ-CoDel). Even though fairness is achieved through the former one, it does not satisfy the real-time video streaming which experience varying RRT and queuing delay. So in this work, Hybrid FQCODEL, which the combination of both the CoDel and Adaptive CoDel with flow is queuing, is proposed for effective reduction of the queuing delay and to increase the throughput of video traffic over the network.

The rest of the paper is organized as follows. Section 2 describes the Bufferbloat problems. Section 3 briefly describes the working of recent $\mathrm{AQM}$ techniques like CoDel with the fair queuing and Adaptive CoDel and a few open issues related to them. Section 4, talk about the Hybrid FQ-CoDel in detail Section 5 consists of the simulation result of the proposed work. Section 6 summarizes the research and concludes the paper with possible future directions.

\section{BUFFERBLOAT}

Bufferbloat is defined as the existence of excessively large (bloated) buffers in systems, particularly network communication systems [9]. Bufferbloat is a phenomenon in packet-switched networks, in which excess buffering of packets causes high latency and packet delay variation (also known as jitter), as well as reducing the overall network throughput. When a router device is configured to use excessively large buffers, even very highspeed networks can become practically unusable for many interactive applications like voice calls, chat, and even web surfing.

Bufferbloat occurs when a network link becomes congested, causing packets to become queued in buffers for too long. In a first-in first-out queuing system, overly large buffers result in longer queues and higher latency, but do not improve network throughput and may even reduce good put to zero in extreme cases. 


\subsection{Cause of Bufferbloat}

Bufferbloat as an issue is caused mainly assumptions and buffering packets for too long in cases where they should be dropped, in an attempt to keep a congested link as busy as possible.

The rule of thumb for the network equipment manufacturers was to provide buffers large enough to accommodate $250 \mathrm{~ms}$ (or more) worth of traffic passing through a device. For example, that way, a router's $1 \mathrm{Gbit} / \mathrm{s}$ Ethernet interface requires a huge $32 \mathrm{MB}$ buffer [11]

Sizing of the buffers according to thumb rule can lead to TCP's congestion-avoidance algorithms causing problems such as high and variable latency, and choking network bottlenecks for all other flows as the buffer becomes full of the packets of one TCP stream and other packets are then dropped [9].

The increase of the round trip time is caused by the buffer on the bottleneck, the maximum increase gives a rough estimation of its size in milliseconds [10].

\section{ACTIVE QUEUE MANAGEMENT}

In Internet routers and other network devices, Active queue management (AQM) is described as the intelligent drop of network packets inside a buffer associated with a network interface controller (NIC) [8], when that buffer becomes full or gets close to becoming full, often with the larger goal of reducing network congestion. This task is performed by the network scheduler, which for this purpose uses various algorithms such as random early detection (RED), Explicit Congestion Notification (ECN), or controlled delay (CoDel). The most recent one among the AQM techniques is CoDel.

\subsection{Random Early Detection}

Random early detection (RED), also known as random early discard or random early drop is a queuing discipline for a network scheduler suited for congestion avoidance. RED mechanism can be useful in controlling the average queue size even in a network where the transport protocol cannot be trusted to be cooperative. RED statistically drops packets from flows before it reaches its hard limit. This causes a congested backbone link to slow more gracefully, and prevents retransmit synchronization. This also helps TCP find its 'fair' speed faster by allowing some packets to get dropped sooner keeping queue sizes low and latency under control [5]. The probability of a packet being dropped from a particular connection is proportional to its bandwidth usage rather than the number of packets it transmits. The main objective of the RED are

- Detect incipient (soon to happen) congestion, allow bursts traffic.

- $\quad$ Keep power (throughput/delay) high by maintaining low average queue size and assume hosts respond to lost packets

- Avoid window synchronization by randomly marking the packets.

- $\quad$ Avoid bias against bursty traffic.

- Provide some protection against ill-behaved users.

\subsection{Controlled Delay Algorithm}

In network routing, CoDel for controlled delay is a scheduling algorithm for the network scheduler. Controlled Delay (CoDel) is the most recent AQM mechanism proposed by Nichols and
Jacobson [7] and is believed to be the best to handle Bufferbloat. Unlike other RED based AQM mechanisms, CoDel is independent of various network parameters such as queue size, queue size averages, queue size thresholds, rate measurements, link utilization, drop rate, queue occupancy time or round trip delays [7].

CoDel relies on the packet sojourn time i.e. the actual queue delay experienced by a packet as a metric to predict congestion in the network. If the packet sojourn time is above the target value for a specified interval of time, CoDel starts proactively dropping/marking the packets to control the queue length.

\subsubsection{Target and parameter}

There are two most important CoDel parameters to be set to achieve optimal results: target and interval. These are fixed parameters and their values are chosen based on the observations from several experiments. Following are the values for target and interval:

- $\quad$ Target $=$ acceptable standing queue delay (constant $5 \mathrm{~ms}$ )

- $\quad$ Interval $=$ time on the order of worst case RTT through the bottleneck (constant between $10 \mathrm{~ms}$ to $1 \mathrm{sec})$

\subsection{Adaptive Controlled Delay}

Adaptive CoDel is used for mitigating the bufferbloat in the network devise and to improve the QoS parameters of the realtime stream. The base of the Adaptive Controlled delay is the varying buffer size needed to support the incoming and outgoing bandwidth for the video traffic over the network. Here the size of the buffer remains same but the queuing delay experienced by each packet in the specified interval gets varies.

Adaptive CoDel helps in refining the fixed target set by the CoDel algorithm to suit the current network parameters. CoDel is independent of any network parameters but the adaptive CoDel rely upon the RTT value for the network traffic which has the changeable nature with each segment [4]. Hence estimation of RRT for each segment is required for adaptive CoDel.

\subsubsection{Target and Interval}

Adaptive CoDel is based on varying RTT, the optimization of bandwidth can be obtained when the buffer size is proportional to the outbound link bandwidth. The actual dispute lies in the determination of proportionality constant. Thus the target value can be given as

$$
\begin{gathered}
\text { Period }=\text { SRTT } \\
\text { Target }=S R T T \text { BWOUT } / \sum B W
\end{gathered}
$$

Where,

- $\quad$ SRTT is the smoothened RTT,

- $\quad$ BWout is the outgoing link bandwidth and $\Sigma B W$ is the sum of all bandwidths in the path of the network flow [4].

\subsection{Limitations of Existing System}

Some of the issues that are present in the existing AQM mechanisms are

- RED algorithm is too difficult to configure especially in an environment with dynamic link rates and it requires manual configuration.

- RED needs to be deployed at the edge of the network. 
- CoDel have fixed target value that does not support real time streaming video.

- CoDel does not perform well with higher number of hops and varying RTT.

- CoDel can be applied to the single queue system as a straight AQM.

- Fairness in network flow is not analyzed in the existing System.

CoDel has implementation advantage over other AQM techniques since the packets get dropped at the dequeue stage. It has got uniform target value to be set for optimal result. But presumptuous of uniform or unique value for the varying input and output bandwidth does not work well. Especially for the real time streaming of video, the uniform target set by the CoDel proves to be insufficient to achieve high throughput.

\section{HYBRID FQ-CODEL}

\subsection{Overview}

According to the limitations of existing AQM mechanisms, we present some solutions to fit it such as fairness and video optimization. The proposed system involves the concept of optimizing the video transmission over the network with reduced queuing delay and improved buffer management. Recent technology for carrying out the above activities especially in mitigating the bufferbloat problem and reduction in queuing delay is some of the prominent AQM mechanism such as Controlled Delay (CoDel) and Adaptive Controlled Delay. These methods have their own advantage based on the specific network environment. The former technique sets a fixed target and the interval time for the packets in the queue, which makes it unsuitable for video streaming. The later one is suitable for real video transmission since it supports the fluctual bandwidth and varying packet size. The fairness of the network flow is yet to be analyzed in the existing system.

Hence there is a need to develop a methodology which supports all types of network traffic so that fairness is achieved with the increased network performance for video transmission. Advantage of this methodology is it provides isolation for low-rate traffic such as DNS, web, and videoconferencing traffic without compromising the benefits of the above mentioned AQM techniques. It improves utilization across the networking fabric and increase the video quality.

\subsection{Methodology}

To solve the aforementioned problem, Hybrid Fair Queuing Controlled Delay (Hybrid FQ-CoDeL) algorithm is developed. Hybrid FQ-CoDel is a combination of both CoDel and Adaptive CoDel AQM technique with Fair Queuing scheduler. Hybrid FQ-CoDel mixes packets from multiple flows and reduces the impact of head of line blocking from bursty traffic [12]. In order to isolate packets form the network flow, a network classifier is used. CoDel is initiated other traffic flow queue, whereas Adaptive CoDel is used in video traffic queue. This method makes use of weighted Deficit Round Robin scheduler algorithm for dequeuing the packets from the buffer of the network devices to the outbound link

\subsubsection{Terminology and concepts}

- Flow: A flow is typically identified by the five tuples of source IP, destination IP, source port, destination port and the protocol. It can also be identified by the subset, superset of those parameters or other means.
- Queue: Queue of packets represented internally in Hybrid FQ-CoDel. In most instances each flow gets its own queue. Queue represents the internal data structure and the flow refers to the actual stream of packets being delivered to the Hybrid FQ-CoDel.

- Classifier: A mechanism to classify the network flow based on the protocol used.

- Scheduler: A mechanisms to select which queue a packet is dequeued from. Here Priority based Deficit Round Robin Scheduler is used

- CoDel and Adaptive CoDel: The Active queue management algorithm employed in Hybrid FQCoDel.

- Quantum: The maximum amount of bytes dequeued from a queue at once.

\subsubsection{CoDel and Adaptive CoDel}

The Hybrid FQ-CoDel combines the function of both the controlled delay and Adaptive controlled delay AQM mechanism. Here the CoDel gets activated in the queue with all other network traffic except the queue with the video data traffic [12]. For the video flow Adaptive CoDel is initiated which proves to be beneficial for the video transmission. But for the low rate traffic CoDel is more sufficient. Thus the benefits of both the AQM mechanisms were used accompanied with fairness queuing.

Here the CoDel have a fixed parameter of 5ms target and 100 millisecond of interval time. When the packets in the queue gets delayed more than $5 \mathrm{~ms}$ then it is dropped at the dequeuing state and the interval time is minimized. By this, the Queuing delay is controlled for the other network traffic.

In the case of video traffic flow, the Adaptive CoDel gets initiated which measures the smoothed RRT for each flow of the video data. It is proved to be beneficial for the real time video streaming and hence the overall network performance increase which helps in video optimization with better quality of video.

\subsubsection{Fair Queuing}

Fair queuing (FQ), also commonly called the fairness algorithm, is a scheduling algorithm that addresses the basic limitation of FIFO queuing. FQ classifies packet flows into multiple queues, offering a fair scheduling scheme for the flows to access the link. In this way, FQ separates traffic and flows, and avoids applications that consume less bandwidth being starved by applications that consume more bandwidth.

Here the classifier is used for separating the traffic into different flow and the higher priority is set for the queue with the video traffic. The scheduler used in Hybrid FQ-CoDel is the priority based deficit round robin which dequeue the packets form the buffer to the outbound link. 


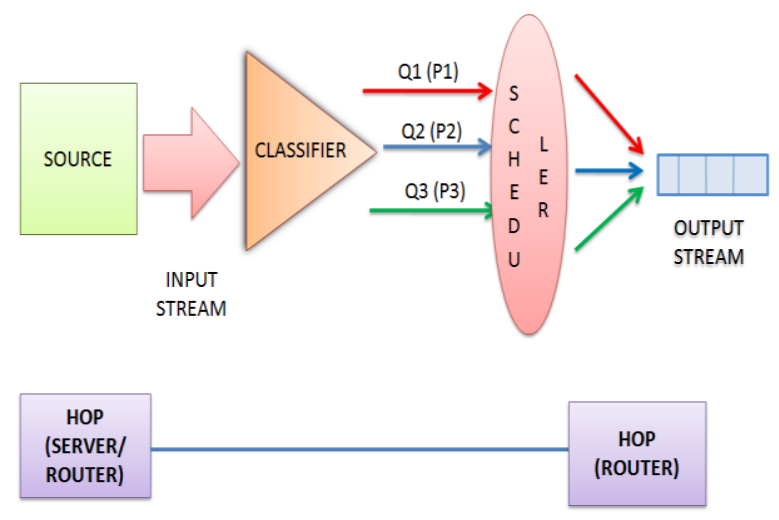

Fig. 1 Architecture of Hybrid FQ-CoDel

The Architecture of the proposed Hybrid FQ-CoDel as shown in fig 1 consists of the following process

- The input stream of data from the source which may be the server or the intermediate network devices gets classified by the network classifier.

- Here, the classifier mechanism help in isolating the generic traffic flow into different kind like video data traffic, DNS, web, and other low rate traffic.

- By analyzing the header of each packet, the classifier will set the priority. In order to optimize video transmission, video traffic is give high priory by the classifier.

- Once the flows are isolated the AQM mechanisms such as CoDel and Adaptive CoDel gets initiated respectively in each flow. In this process only the flows with video traffic will have Adaptive CoDel mechanisms for maintaining queuing delay where as others will have CoDel running in them simultaneously.

- In order to achieve fairness in resources utilization, Scheduler has been used which select, which queue a packet is dequeued from. Here Priority based Deficit Round Robin Scheduler is used

\section{SIMULATION RESULTS}

In order to evaluate the performance of Hybrid FQ-CoDel algorithm, we also implemented the approach proposed by [3] in module 1.Fig. 2 shows the CoDel Average queue size rate versus the time. In Fig. 3 the Average queue size versus the time for the proposed Hybrid FQ-CoDel is shown, which indicate the reduced Queuing Delay for the packet flow.

Fig. 4 shows the comparison between the proposed AQM algorithms Hybrid FQ-CoDel with the existing AQM mechanism in terms of the Queue length for the maximum resource utilization which in turn provides the comparison of the queuing delay.

Because of the use of both the CoDel and Adaptive CoDel with the mechanism of the Scheduler the queuing delay of the overall network is reduced which also reduces the Bufferbloat problem.

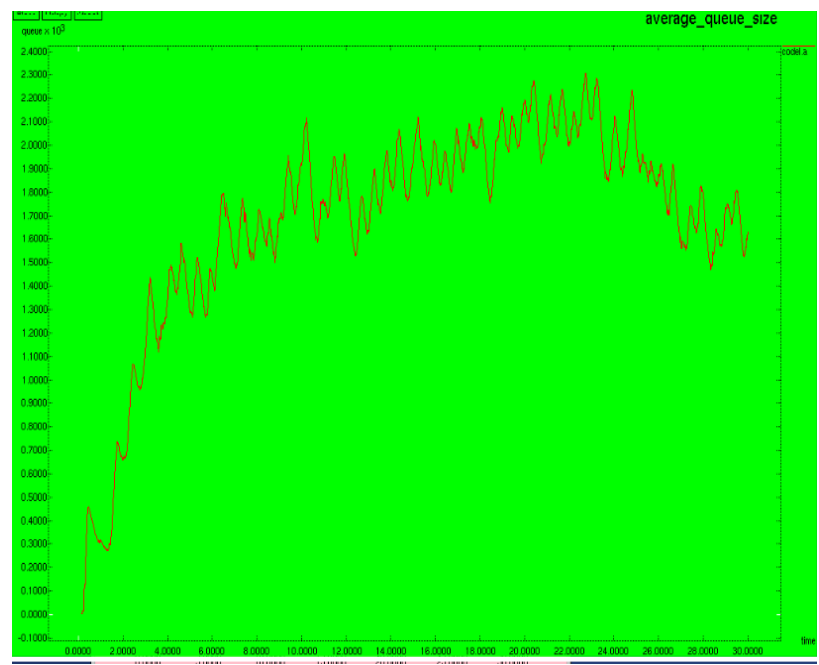

Fig. 2 Average Queue Length vs Time for CoDel

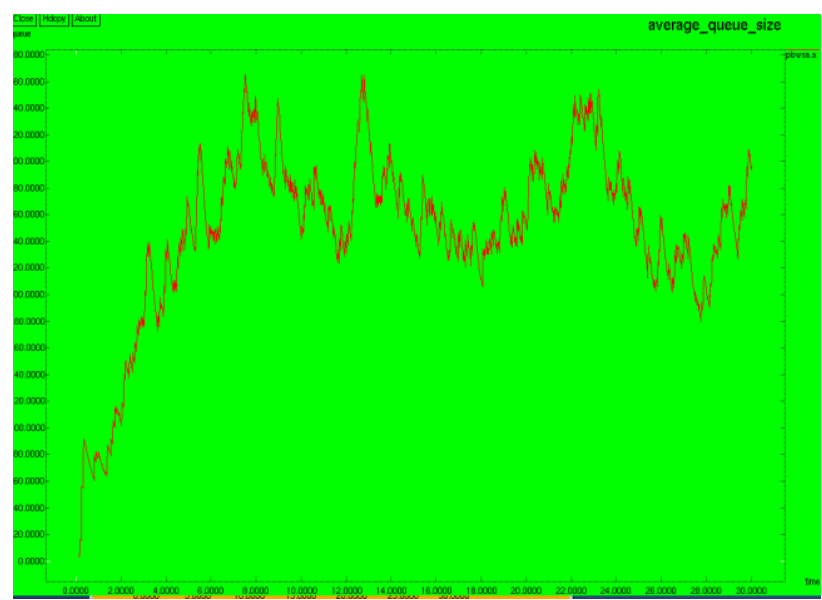

Fig. 3 Average Queue Length vs Time for Hybrid FQCoDel

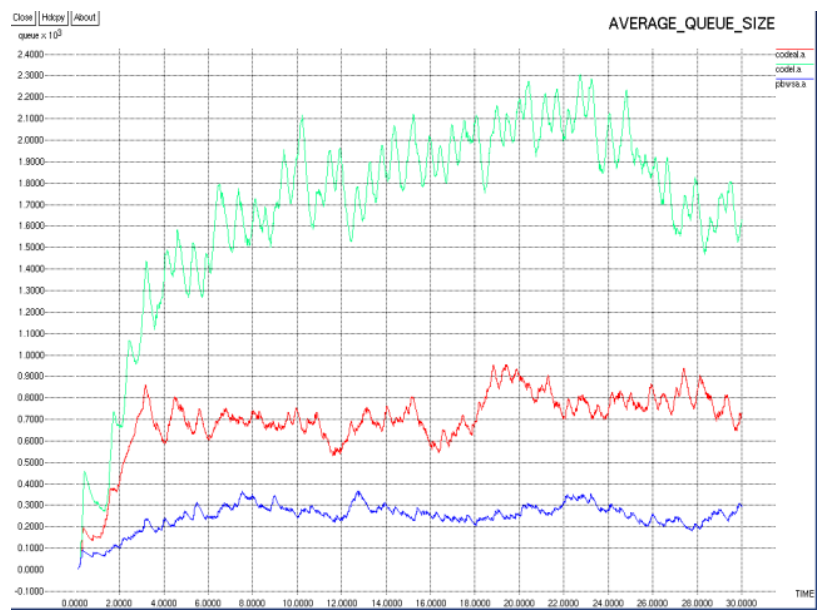

Fig. 4 Comparison of Hybrid FQ-CoDel with other AQM Algorithm

\section{CONCLUSION}

In this paper, a new AQM mechanism called Hybrid FQ-CoDel for efficient video transmission over the network is proposed Here the proposed Hybrid FQ-CoDel algorithm efficiently reduces the queuing delay and also mitigates the bufferbloat problem that exits in the network devices. By reducing the 
delay the propose approach can enhance the network performance. Simulation results shows that the Hybrid FQCoDel have reduced Queuing Delay than the existing system which also helps to reduce bufferbloat problem. For future work may include the implementation of the above proposed work, in adapting different usage of scheduling algorithm with different network environment for reducing queuing delay.

\section{REFERENCES}

[1] YasharGanjaliGavgani, March 2007, "Buffer Sizing in Internet Routers" Ph.D, dissertation Stanford University, California.

[2] J. Gettys and K. Nichols, "Bufferbloat: Dark Buffers in the Internet,"Communications of the ACM, vol. 55, no. 1 , pp. 57-65, 2012.

[3] K. Nichols and V. Jacobson, "Controlling Queue Delay," Communications of the ACM, vol. 55, no. 7, pp. 42-50, 2012.

[4] Matilda.S, B.Palaniappan, Thambidurai.P, "Bufferfloat Mitigation for Real-time Video Streaming using Adaptive Controlled Delay Mechanism”, International Journal of Computer

[5] S. Floyd and V. Jacobson, "Random early detection gateways for congestion avoidance", IEEE/ACM Transaction on Networking, Vol.1, No. 4, pp. 397-413,
1993.Applications (0975 - 8887) Volume 63 - No. 20, February 2013

[6] Guerin, R.Peris, V: Quality-of-Service in Packet Networks: Basic Mechanismsand Directions. Computer Networks, Vol. 31, 1999, pp. 169-189.

[7] K. Nichols and V. Jacobson, "Controlling Queue Delay," ACM Queue Magazine: Networks, vol. 10, no. 5, pp. 6881, May 2012.

[8] Labrador, M.Banerjee, S.: Packet Dropping Policies for ATM and IP Net-works. IEEE Communications Surveys, Vol. 2, 1999, No. 3, pp. 2-14.

[9] Gettys, Jim, "Bufferbloat: Dark Buffers in the Internet". IEEE Internet Computing, vol. 15 (3).IEEE. pp. 9596 Retrieved 2012-02-20.

[10] Guido Appenzeller; Isaac Keslassy; Nick McKeown (2004). "Sizing Router SIGCOMM.,ACM.Retrieved 2013-10-15.

[11] Clunis, Andrew, "Bufferbloat demystified". Retrieved 2013-09-27.

[12] Vimaladevi. V, Manju Bala. P, "Video Optimization through Hybrid FQ-Codel Algorithm", International Journal of Scientific Research in Science, Engineering and Technology (IJSRSET), Volume 1, Issue 6, pp.377381, November-December-2015. 